\title{
Structural Determinant of Protein Designability
}

\section{Citation}

England, Jeremy L., and Eugene I. Shakhnovich. 2003. "Structural Determinant of Protein Designability." Physical Review Letters 90 (21). https://doi.org/10.1103/physrevlett.90.218101.

\section{Permanent link}

http://nrs.harvard.edu/urn-3:HUL.InstRepos:41417287

\section{Terms of Use}

This article was downloaded from Harvard University's DASH repository, and is made available under the terms and conditions applicable to Other Posted Material, as set forth at http:// nrs.harvard.edu/urn-3:HUL.InstRepos:dash.current.terms-of-use\#LAA

\section{Share Your Story}

The Harvard community has made this article openly available.

Please share how this access benefits you. Submit a story.

Accessibility 


\title{
Structural Determinant of Protein Designability
}

\author{
Jeremy L. England and Eugene I Shakhnovich* \\ Harvard University, Department of Chemistry and Chemical Biology, \\ 12 Oxford Street, Cambridge, Massachusetts 02138
}

(Dated: October 27, 2018)

\begin{abstract}
Here we present an approximate analytical theory for the relationship between a protein structure's contact matrix and the shape of its energy spectrum in amino acid sequence space. We demonstrate a dependence of the number of sequences of low energy in a structure on the eigenvalues of the structure's contact matrix, and then use a Monte Carlo simulation to test the applicability of this analytical result to cubic lattice proteins. We find that the lattice structures with the most lowenergy sequences are the same as those predicted by the theory. We argue that, given sufficiently strict requirements for foldability, these structures are the most designable, and we propose a simple means to test whether the results in this paper hold true for real proteins.
\end{abstract}

PACS numbers:

Successful protein design relies in part on knowledge of how a polypeptide chain's native structure varies as a function of its amino acid sequence. Yet even if this "protein folding problem" were solved in its entirety, the would-be protein designer would still face the formidable task of finding those sequences which folded to the target structure he or she wished to engineer [1, 2]. It is therefore vital to know how many solutions to this search of sequence space exist for a given target structure, i.e. how designable the target structure is [2, 3, 4, 5, 6, 7]. The question of what makes a particular protein structure more designable also bears fundamentally on our understanding of how proteins first evolved.

Past studies of designability have been limited in large part by their lack of generality. The previous contributions in 4, 5], for example, rely heavily on their study of two-letter monomer alphabets and Cartesian latticepolymers, and can therefore offer no clear implications for the 20-letter, off-lattice world of real proteins 2]. In contrast, studies like that of Koehl and Levitt 7] come closest to probing real protein designabilities, but have no theoretical foundations from which to extrapolate beyond their numerical results. Finally, a number of investigations have assumed that the distribution of amino acid sequence energies is either nearly [ 8$]$ or totally $[\underline{6}]$ independent of the structural topology of the target fold, a premise which is flatly contradicted by the findings in 5 . The intention of this communication is therefore to identify a theoretically motivated, generally applicable quantitative measure of structural topology which we expect to be a good predictor of designability.

In this Letter, we develop an approximate analytical theory of the spectrum of possible monomer sequence energies for a heteropolymer in any given conformation. Our theory leads us to identify a novel topological property of a conformation, its contact trace, as a determinant of the shape of the conformation's sequence spectrum. We then confirm the predicted usefulness of the contact trace by running Monte Carlo simulations under condi- tions which are more realistic than those for which the contact trace was originally identified. Finally, we use the results of these simulations to suggest a connection between the contact trace and designability, and we propose a way to test this hypothesis on real protein structures.

As a preliminary, we must draw a distinction between what we term "strong designability" and "weak designability". We define a structure's "strong designability" in the same way that previous studies have defined its "designability": as the total number of amino acid sequences that fold to that structure. Thereotical arguments based on the random energy model suggest that in order for a sequence to be foldable, its native state energy must lie below a certain low-energy threshold $E_{c}$ 5, 9, 10, 11, 12], which represents the lowest energy range accessible to misfolded conformations. We therefore define a structure's "weak designability" to be the fraction of its sequences which lie below a low energy cutoff $E_{c}$. It is important to note that though the strong and weak versions of designability are set apart a priori, it is possible that they will turn out to be the same thing in practice. Indeed, we will argue later on that if natural thermodynamic and kinetic requirements for folding are sufficiently stringent, then a structure's strong and weak designability become essentially indistinguishable.

Our first aim is to determine whether contact topology effects a structure's distribution of possible sequence energies, and to this end we derive a closed-form sequence partition function for a special class of amino acid alphabets. We begin by considering a polymer of $N$ monomers, where each monomer can be one of $2 M$ different possible kinds. We may construct for any polymer configuration an $N \times N$ traceless contact matrix $C$ whereby lattice nearest neighbors that are not sequence neigbors are considered to be in contact [13]. Next, we represent the amino acid type of the $i$ th monomer in the chain as a $2 M$ dimensional unit-vector $\vec{s}^{(i)}=[0, \ldots, 0,1,0, \ldots, 0]$, where the non-zero vector element is the $k$ th element of the vector if the $i$ th monomer is of type $k$. 
We define the Hamiltonian to be a standard nearestneighbor contact potential, i.e.

$$
\mathcal{H}=\frac{1}{2} \sum_{i, j}^{N, N} C_{i, j} \vec{s}^{(i)} \cdot\left(B \vec{s}^{(j)}\right)
$$

where $B$ is the $2 M \times 2 M$ matrix of interaction energies for the different pairs of monomer types. A closed form expression for the partition function of this Hamiltonian may be obtained if we let $B$ take on the special form

$$
B=\left[\begin{array}{ccccc}
V_{1,1} & -V_{1,1} & V_{1,2} & -V_{1,2} & \cdots \\
-V_{1,1} & V_{1,1} & -V_{1,2} & V_{1,2} & \cdots \\
\vdots & \vdots & \vdots & \vdots &
\end{array}\right]
$$

In other words, $B$ is the direct product of a matrix $V$ with an Ising-like potential. Though we have now constrained the nature of our contact potential, we still retain unlimited freedom to choose the types of interactions represented in $V$.

Now let us define the $M$-vector $\vec{\sigma}$ through $\sigma_{k+1}=$ $s_{2 k+1}-s_{2 k+2}$. Our Hamiltonian becomes

$$
\mathcal{H}=\frac{1}{2} \sum_{i, j}^{N, N} C_{i, j} \vec{\sigma}^{(i)} \cdot\left(V \vec{\sigma}^{(j)}\right)
$$

where $\vec{\sigma}^{(i)}$ is a vector of unit length whose single non-zero element may be either 1 or -1 .

At this point we define the $M N \times M N$ block matrix $\mathbf{U}$ through $\mathbf{U}_{M(i-1)+k, M(j-1)+l}=C_{i, j} V_{k, l}$. In other words, we turn every element of $C$ into an $M \times M$ block which couples the vectors $\left\{\vec{\sigma}^{(i)}\right\}$ through $V$ wherever there is a contact. Finally, if we write $\boldsymbol{\sigma} \equiv\left[\vec{\sigma}^{(1)}, \ldots, \vec{\sigma}^{(N)}\right]$ then the Hamiltonian can be expressed as

$$
\mathcal{H}=\frac{1}{2} \sigma \cdot(\mathbf{U} \boldsymbol{\sigma})
$$

This form of the Hamiltonian will allow us to calculate the sequence space partition function, for any contact map and any potential matrix $V$. To do so, we employ a continuous-spin approximation, allowing each vector $\vec{\sigma}^{(i)}$ to swing anywhere on the $M$-dimensional unit sphere instead of restricting it to one of the $2 M$ available unit-integer lattice points. This approximation not only smears the discrete sequence spectrum into a continuous one, but also distorts the spectral width by altering the relative sizes of $M$ and $M-1$ letter sequence spaces, thereby preventing the theory from making quantitatively accurate predictions. Our assumption is that the model nevertheless retains important information about the effect that variations in contact topology have on the shape of the spectrum. The partition function now becomes

$Z(\beta)=\int d^{M N} \boldsymbol{\sigma}\left(\prod_{i=1}^{N} \delta\left(\left|\vec{\sigma}^{(i)}\right|-1\right)\right) \exp \left[-\frac{\beta}{2} \boldsymbol{\sigma} \cdot(\mathbf{U} \boldsymbol{\sigma})\right]$
Assuming hereafter that $M \gg 1$, we have

$$
d^{M} \vec{\sigma} \exp \left[-\frac{(M-1)}{2}|\vec{\sigma}|^{2}\right] \simeq d|\vec{\sigma}| \delta(|\vec{\sigma}|-1)
$$

Defining $\mathbf{M}=(M-1) \mathbf{I}$, where $\mathbf{I}$ is the $M N \times M N$ identity matrix, and $\mathbf{u}=\mathbf{M}^{-1} \mathbf{U}$ we may write

$$
Z(\beta)=\int d^{M N} \boldsymbol{\sigma} \exp \left[-\frac{1}{2} \boldsymbol{\sigma} \cdot(\mathbf{M}+\beta \mathbf{U}) \boldsymbol{\sigma}\right]
$$

and, since $\mathbf{U}$ is a real symmetric matrix, we finally obtain

$$
\frac{Z(\beta)}{Z(0)} \equiv z(\beta)=\sqrt{\frac{\operatorname{det}[\mathbf{M}]}{\operatorname{det}[\mathbf{M}+\beta \mathbf{U}]}}=\frac{1}{\sqrt{\operatorname{det}[\mathbf{I}+\beta \mathbf{u}]}}
$$

If we use $\operatorname{det} \mathbf{A}=\exp \operatorname{Tr} \ln \mathbf{A}$ to expand $\ln z$ to $\mathcal{O}\left(\beta^{2}\right)$ and inverse-Laplace transform the resulting approximation of the partition function to obtain a distribution of sequence energies $n(E)$, we get

$$
\begin{aligned}
n(E) \approx \frac{1}{2 \pi} \int e^{i \beta E} z(i \beta) d \beta & \simeq \exp \left[-\frac{E^{2}}{\operatorname{Tr} \mathbf{u}^{2}}\right] \\
& \simeq \exp \left[-\frac{E^{2}}{2 N_{C} \sigma_{B}^{2}}\right]
\end{aligned}
$$

where $N_{C}$ is the number of contacts and $\sigma_{B}^{2}$ is the variance of the interaction energies in $B$. A similar result was obtained in 2] by a straightforward expansion of the sequence partition function. Equation (9) says that the Gaussian approximation of the density of states leads to a naïve sequence space random energy model (REM) for $n(E)$. It is this REM picture, in which all structures with the same number of contacts have identical sequence spectra, which has been used implicitly in [6]. We demonstrate below, however, that the consideration of higher order terms in the expansion of $\ln z$ can have a profound impact on how we understand designability.

Let us now consider the free energy $F=-\frac{1}{\beta} \ln z$ of our sequence space partition sum. Defining the matrix $v=\frac{1}{M-1} V$ and expanding about high "design temperature" (i.e. requiring that $\left|\beta \lambda_{i}\right|<1$, for all $\lambda_{i}$ which are eigenvalues of $\mathbf{u}$ ), we get

$$
\begin{array}{r}
F=-\frac{\beta}{4}\left(\operatorname{Tr} v^{2}\right)\left(\operatorname{Tr} C^{2}\right)+\frac{\beta^{2}}{6}\left(\operatorname{Tr} v^{3}\right)\left(\operatorname{Tr} C^{3}\right) \\
-\frac{\beta^{3}}{8}\left(\operatorname{Tr} v^{4}\right)\left(\operatorname{Tr} C^{4}\right)+\mathcal{O}\left(\beta^{4}\right)
\end{array}
$$

Those structures which minimize $F$ will be the ones with the greatest number of amino acid sequences that have low energy when threaded onto that structure. Minimization of $F$ is therefore a means to maximize weak designability, which we recall is determined by the fraction of low-energy sequences in a structure's sequence spectrum. Thus, we now consider which choices of the 


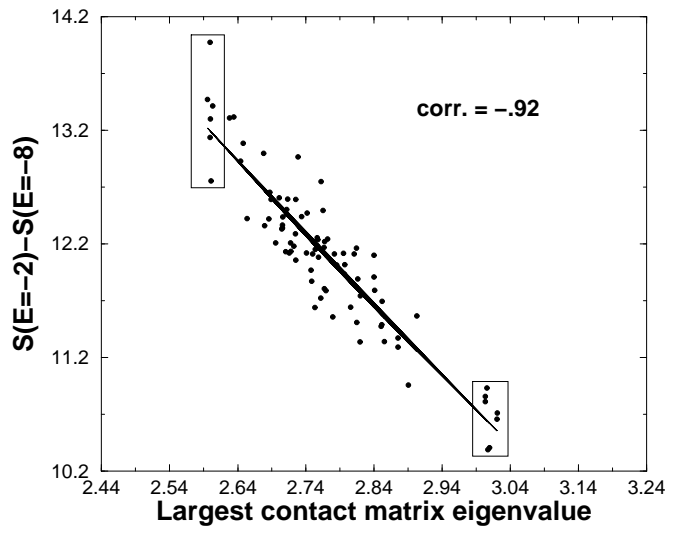

FIG. 1: The difference in sequence space entropy between an energy near the peak of all structural sequence spectra $(E=-2)$ and one in the lower tail of all spectra $(E=-8)$ as a function of the contact trace, measured here by the largest eigenvalue of the structure's contact matrix (which follows from $\operatorname{Tr} C^{n}=\sum \lambda_{i}^{n}$ ). Each point was generated from data collected while slowly annealing a Monte Carlo sequence design simulation from high temperature $(T=2)$ to low $(T=.2)$, with $10^{7}$ Monte Carlo steps taken at each temperature. The boxed points correspond to structures which were chosen by hand so as to ensure that the extrema of the range of possible eigenvalues were represented. All other structures were chosen randomly.

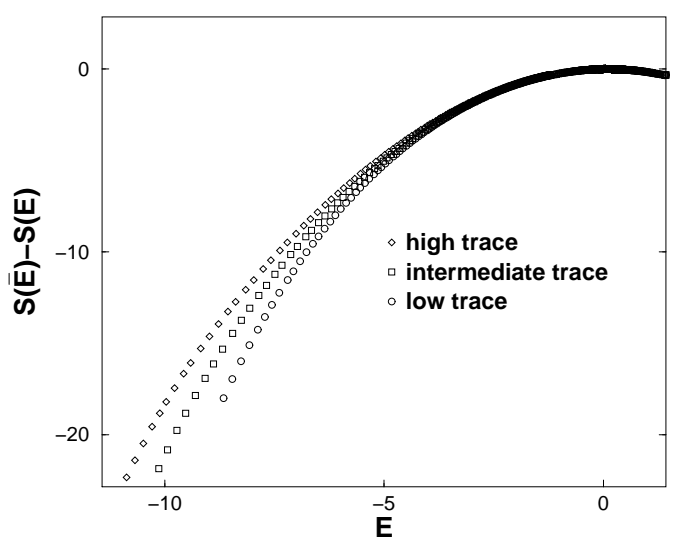

FIG. 2: The change in sequence space entropy from energy $\langle E\rangle(T=\infty)=\bar{E}$ to energy $E$ for three structures with largest contact matrix eigenvalues of 3.02 (high trace), 2.78 (intermediate trace), and 2.60 (low trace).

contact matrix $C$ would serve to make the free energy $F$ as negative as possible. Because $\operatorname{Tr} C^{n}$ is equal to the number of $n$-step paths along the contact map which return to their starting place, we know that all such contact traces must be positive. Thus, the exact behavior of the series in (10) will hinge on whether the largest eigenvalues of $v$ are positive or negative.

For either type of potential matrix $v$, however, we expect that there will be some positive correlation between the trace of an even power of a structure's contact matrix and the number of low energy monomer sequences in that structure. Furthermore, the dependence of the free energy expansion in (10) on such coarse quantities as the traces of powers of $v$ suggests that the impact of the contact matrix on the spectrum of sequence energies should be relatively insensitive to the detailed features of the potential. We therefore determined to empirically test whether the above results remained valid for a discrete monomer alphabet which violated the special form of the potential assumed in (2). We first calculated the contact matrices for all 103346 different compact conformations of 27-mers on a cubic lattice 13. Next, we calculated $\langle E\rangle$ vs. $T$ annealing curves for random starting sequences on different structures for a standard Monte Carlo search of sequence space with a move set containing compositionpreserving two-monomer and three-monomer permutations. The energy of each sequence was determined using a potential set given by Table 6 of [14]. This set of interactions, where average interactions are subtracted out, is one of the most diverse potentials possible for a 20 letter alphabet, and therefore provides the most general empirical test of the predicted relationship between sequence energies and contact topology. From the annealing curves, we then calculated the entropy in sequence space $S(E)$ according to the prescription given by eq. (11) of [2].

Figure 1 plots the sequence space entropy difference between low and near-modal energy versus the largest eigenvalue of the structure's contact matrix for 86 randomly-selected lattice structures. As predicted, the entropy difference between the peak and the left tail decreased as the largest contact matrix eigenvalue increased (corr. $=-0.92$ ), indicating that more sequences have low energy in high trace structures. Figure 2 illustrates that the effect observed in Fig. 1 results from global differences in the shapes of the sequence spectra of high trace and low trace structures. The higher the contact trace, the more gradually the number of sequences falls off as energy decreases, and therefore the greater the relative number of sequences of low energy. Clearly, the contact trace of the target structure controls how low in energy a Monte Carlo sequence optimization algorithm running at fixed temperature $T_{\text {des }}$ will be able to search. The greater the contact trace, the larger the $S(E)$ at low energies, i.e. the greater the weak designability.

Interestingly, the most designable 27-mer structures identified using our maximum eigenvalue determinant are similar to the one identified in 15] using random sampling of sequences and a different, "solvation-like" Miyazawa- Jernigan potential. This attests to a generality of our proposed structural determinant of designability with respect to potentials.

Structures of high contact trace are weakly designable, but are they strongly designable? In order to 


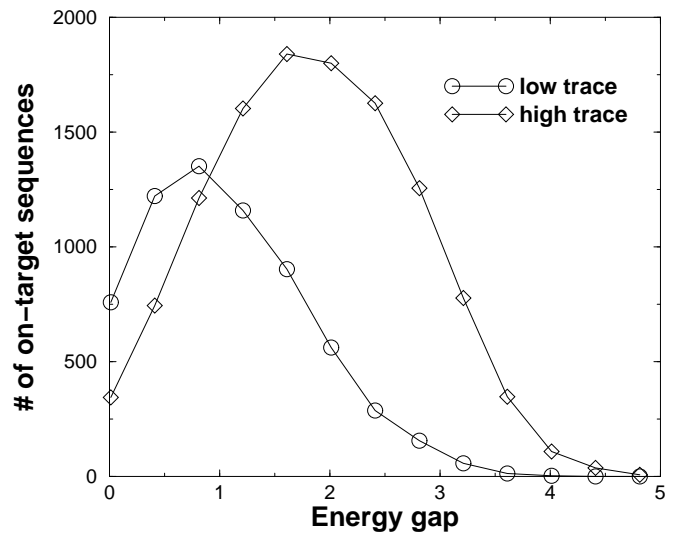

FIG. 3: We designed sequences on two different target structures in Monte Carlo searches of $2 \times 10^{7}$ steps $(.2<T \leq .4)$, sampled at every one sequence in $10^{4}$. Here, we plot the number of sequences whose unique compact ground states were the structure on which they were designed, as a function of the difference in energy between the sequence's ground state and the lowest-lying excited state which shares fewer than $25 \%$ of its contacts with the ground state.

address this question, we examined the stability of sequences designed on two structures of maximal and minimal contact trace. For each target structure, we determined how many of its designed sequences were "ontarget", that is, had the target structure as their unique energetic ground state determined over all compact conformations, and calculated the gap in energy between that of the ground state and those of the lowest-energy structures with low structural similarity to the ground state conformation. We found that while only $12 \%$ of the sequences designed for the low trace structure were on-target, $24 \%$ were in the case of the high trace structure. Furthermore, as Figure 3 illustrates, the sequences designed successfully on the high trace structure tended on average to have larger energy gaps than their low trace cousins, consistent with earlier observations that low energy in the native conformation also leads on-average to a larger gap and greater stability [16, 17]

Fig. 3 therefore gives us a means to unify strong and weak designability. Past studies have suggested that in order for a protein sequence to fold rapidly to its native state, it must have a larger-than-average energy gap 12, 18, 19]. Fig. 3 demonstrates that if the conditions for folding stably and quickly in nature demand sufficiently high energy gaps, weak designability will be one and the same with strong designability, since only by having very low energy in its target structure will a sequence have an appreciable chance of exhibiting the gaps which are thermodynamically and kinetically required by the natural environment. We speculate that protein evolution under such conditions would lead to the emergence of natural protein folds with near-optimal contact traces.
We have presented an analytical theory which identifies the first instance of a generally applicable, welldefined, numerical measure of a protein structure's topology which is expected to correlate with the structure's designability. Using a Monte-Carlo search in the sequence space of a lattice model with 20-letter energetics, we have shown that the implications of the theory extend beyond the special assumptions under which they were first developed. The finding that higher contact trace may lead to greater potential for thermal stability leads us to hypothesize that thermophilic organisms, whose natural environment makes uncommonly strict demands for protein stability, exhibit a genomic bias towards folds of higher contact trace. We have recently found that this bias exists (unpublished results), providing further encouragement that the contact trace may yet offer new insight into the laws governing structural diversity in natural proteins.

We thank Edo Kussel, William Chen, Nikolay Dokholyan, and Lewyn Li for valuable discussions, and NIH and Pfizer Inc. for support.

* Electronic address: eugene@belok.harvard.edu

[1] B.Dahiyat and S.Mayo, Science 278, 82 (1997).

[2] E. Shakhnovich, Folding \&Design 3, R45 (1998).

[3] A.V.Finkelstein, A.Gutin, and A.Badretdinov, FEBS Lett. 325, 23 (1993).

[4] H. Li, N. Wingreen, and C. Tang, Science 273, 666 (1996).

[5] E. Kussell and E. Shakhnovich, Phys Rev Lett. 83, 4437 (1999).

[6] N. Buchler and R. Goldstein, J. Chem. Phys, 112, 2533 (2000).

[7] P. Koehl and M. Levitt, Proc. Natl. Acad. Sci. USA 99, 1280 (2002).

[8] G. Tiana, R. Broglia, and D. Provasi, Phys. Rev. E 64, 011904 (2001).

[9] E. Shakhnovich and A. Gutin, Nature 346, 773 (1990).

[10] J. Bryngelson and P. Wolynes, Proc.Natl.Acad.Sci.USA 84, 7524 (1987).

[11] A.V.Finkelstein, A. Gutin, and A. Badretdinov, Proteins 23, 142 (1995).

[12] A. Sali, E. Shakhnovich, and M. Karplus, Journ. Mol. Biol. 235, 1614 (1994).

[13] E. Shakhnovich and A. Gutin, J.Chem.Phys 93, 5967 (1990)

[14] S. Miyazawa and R. Jernigan, Macromolecules 18, 534 (1985).

[15] H. Li, C. Tang, and N. Wingreen, Proteins 49, 403 (2002).

[16] E. Shakhnovich and A. Gutin, Proc Natl Acad Sci USA 90, 7195 (1993).

[17] V. Abkevich, A. Gutin, and E. Shakhnovich, J Chem Phys 101, 6052 (1994).

[18] R. Goldstein, Z. Luthey-Schulten, and P. Wolynes, Proc. Natl. Acad. Sci. USA 89, 4918 (1992).

[19] S. Sato, S. Xiang, and D. Raleigh, J. Mol. Biol. 312, 569 
(2001). 